\title{
An Innovative Technique to Increase Lift of a Coanda UAV
}

\author{
Maliheh Najafi ${ }^{1}$, Mohsen Jahanmiri ${ }^{2}$ \\ ${ }^{\prime}$ (M.E.Graduate, Dept. of Mechanical \& Aerospace Engineering, Shiraz Univ. of Tech.,Shiraz, Iran) \\ ${ }_{2}^{2}$ (Associate Professor, Dept. of Mechanical \& Aerospace Engineering, Shiraz Univ. of Tech.,Shiraz, Iran)
}

\begin{abstract}
In this study, the effect of rotating vanes, installed on the body of the Coanda UAV is investigated both experimentally and numerically, with the aim of improving its aerodynamics performance. By constructing and testing two models of Coanda UAVs with and without axial vanes, for measuring the lift and dynamic pressure distribution on the body, 16\% increase in lift is observed. In addition, a finite volume solver is utilized to investigate the flow over Coanda UAVs with and without rotating vanes. Numerical results show that the installed axial vanes on the Coanda UAV leads to increasing lift about 21\%. The numerical results are in good agreement with the experiments. Overall, the results show that mounting axial vanes on the body of Coanda UAV increase lift. The consequences of installing an axial vane at the separation point of the fuselage surface on the overall lift of the Coanda UAV are discussed.
\end{abstract}

Keywords: Coanda effect, Experimental method, Numerical solutions, Unmanned aerial vehicle

\section{Introduction}

Nowadays, UAVs are very popular aircrafts because of their undeniable advantages such as small sizes, easy and cheap manufacturing, long endurance, lower cockpit errors and more safety. They have a variety of applications in the telecommunications, navigation, meteorological and geological researches, firefighting, and search and rescue. Among all types of UAVs, VTOLs have a special importance because of capability to hover, takeoff and land vertically. Coanda UAV is a brilliant VTOL which uses Coanda effect to produce lift. The essential merits of the Coanda UAV are simple control mechanism and having no external rotating components as a result less vulnerability when crashing.

Coanda UAV generates lift in two ways. Firstly, redirecting flow in downstream region and creating a vertical thrust. Secondly, by routing flow over the surface of the fuselage that alters the pressure field over and below the body. In this UAV, Coanda effect produces lift as long as flow adheres to the surface [1].

When an object rotates in a fluid, for instance, a circular cylinder, the velocity of fluid flow at one side is more than the other side. According to Bernoulli's equation by increasing velocity, the pressure decreases. Thereby this non-symmetrical pressure field applies a force perpendicular to the direction of the fluid flow on the body (Fig. 1). A German physicist, namely Magnus described it, and then it is called Magnus effect [2]. The formulation of this phenomenon was introduced later. Kutta-Joukowski Lift Theorem is one of the most fundamental theories to calculate the lift of any two-dimensional body. This theory was developed by kutta (1902) in Germany andJoukowski (1906) in Russia independently. Lift per unit span of the circular cylinder is perpendicular to the velocity, and is,

$$
L=\rho G V
$$

where, $\rho$ and $\mathrm{V}$ are the density and the free stream velocity respectively, and $\mathrm{G}$ is the circulation that is produced by the rotating cylinder,

$$
G=4 \pi^{2} r^{2} S
$$

$\mathrm{S}$ is the cylinder spin (revs/sec) around its longitudinal axis, and $\mathrm{r}$ is the radius of the cylinder [3].

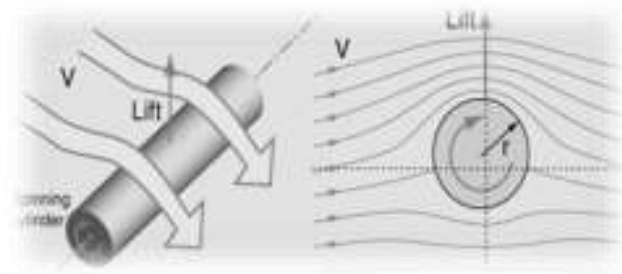

Fig.1Streamlines around a rotating circular cylinder.

The first Coanda UAV was designed by Romanian inventor Henri Marie Coanda in 1932 [4]. Mirkov and Rasuo investigated several Coanda VTOLs with and without active flow control including suction and blowing numerically [5]. Dumas and et al. examined the influence of temperature on Coanda effect numerically. 
Constant (thermal diffusivity) and variable Parndtl numbers were considered. It is reported that raising thermal conductivity postpones flow separation [6]. It is showed that the optimal shape for Coanada UAV is a simple circular section [7]. One of the most well-known Coanda UAVs was developed by Geoff Hatton which is named GFS (Geoff's Flying Saucer) UAV [8]. The flow field around this UAV was simulated numerically by Chen and Sung in 2008. The impact of adding cowling was examined on GFS UAV. It was found that cowling has little positive or even adverse contribution to lift [9]. Zhang and et al. reviewed history and evaluated the hover efficiency of GFS UAV through CFD. A number of cowlings were compared. It is discovered that cowling affects propulsion efficiency on the one hand by jamming exist flow from duct fan negatively, but on the other hand by reducing the overall speed of system acceptably. All in all cowling has an appropriate contribution to the whole vehicle [10].

The purpose of this paper is investigating the consequences of installing an axial vane at the separation point of the fuselage surface on the overall lift of the Coanda UAV. The geometry of the UAV is similar to Naudin No1 project [11] (Fig. 2).

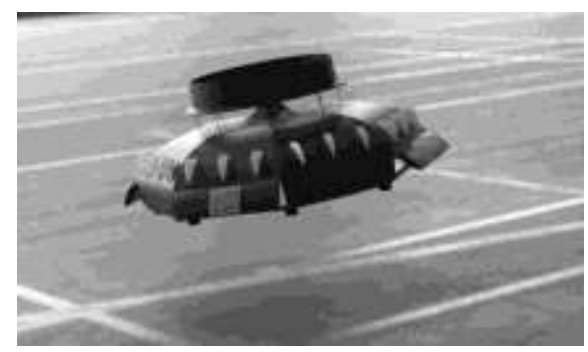

Fig.2 NO1 Coanda UAV.

\section{Experimental Study}

Coanda effect is an elusive phenomenon and depends on many factors therefore; the most optimal geometry of Coanda UAVs has not been introduced yet. In this research, the No1 geometry is opted to implement the new idea to enhance Coanda UAVs lift (Fig. 3). A duct fan blows air toward the curved body, passing air nearby this curved fuselage generates lift on account of the Coanda effect. Pressure in experimental tests and numerical simulations is measured on the curved part of the fuselage (Fig. 3). As it is clear from Fig. 4, a four blade axial vane with 30 degrees tilt angle is mounted on the body at the separation point. The detached flow rotates this vane.

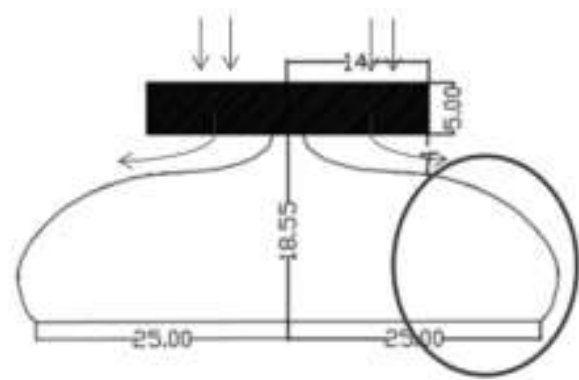

Fig.3 The curvature of the fuselage No1 UAV (dimensions in $\mathrm{cm}$ ).
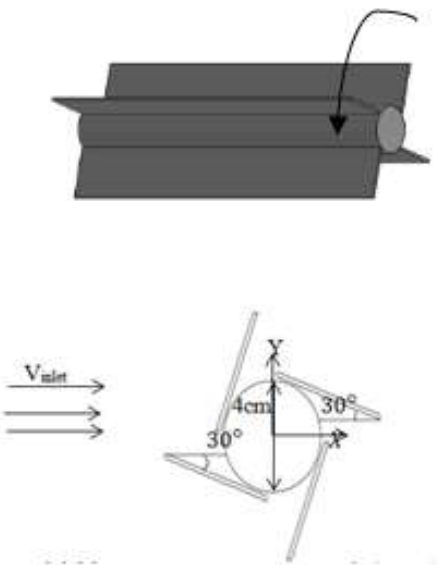

Fig.4 A view of the vane blades. 
The separation point on the surface of the body is determined by tufts flow visualization technique. Shaking or the tendency of tufts to go up from the surface is an indicative of the boundary layer separation [12].

When the rotating speed of the motor was $122 \mathrm{rev} / \mathrm{s}$, the separation point has been distinguished by means of flow visualization subsequently, axial vane set at this location. These steps are illustrated in Fig. 5. Figure 6 demonstrates the model with the axial vane.

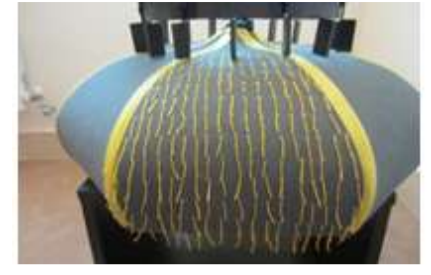

a) Tufts flow visualization technique

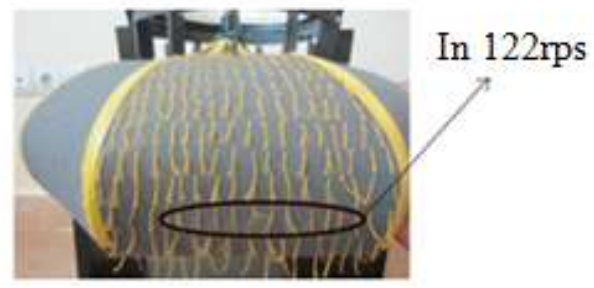

b) Flow separation point

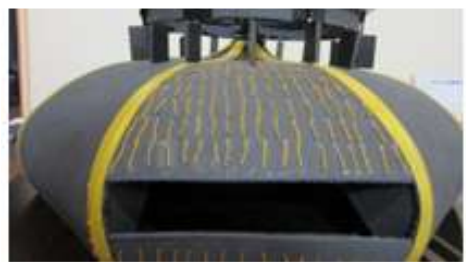

c) The model prior to installing the axial vanes

Fig.5Vans position scheme.

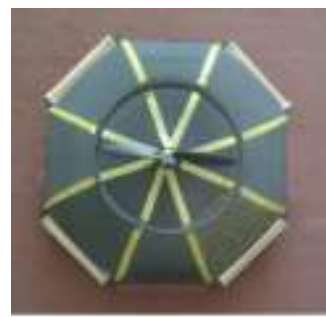

Fig.6 View of model with axial vanes.

\section{Experimental Tests}

Two main aerodynamic characteristics that are considered in this project are lift and dynamic pressure distribution on the body.In spite of the fact that experimental tests were conducted in a situation where the UAV was stabilized and do not turned around its vertical axis by anchoring it from four sides to prevent turning it around vertical axis in opposite direction of the rotor due to the torque, it was located on the ball bearing which allows it to rotate freely around its vertical axis as a precaution to avoid damaging of the motor and fuselage. SF-400 digital balances with $0.01 \mathrm{gr}$ accuracy and $5 \mathrm{~kg}$ capacity were utilized to calculate the lift. A laser tachometer with the ability to measure up to $200 \mathrm{rev} / \mathrm{s}$ was set on the top of the UAV to record the rotation speed of the propeller. Figure 7 shows lift measurement mechanism. To minimize ground proximity interference, the UAV was located on a rigid rod far enough from the ground as displays in Fig. 7.

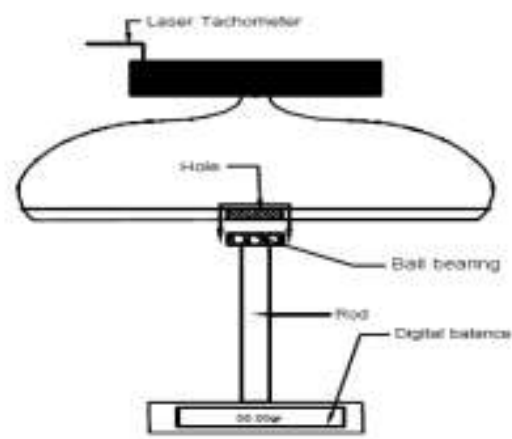

Fig.7 Lift measurement device. 
The followings explain the process of tests. First, the weight of UAV when it is off is measured. Second, the engine is switched on subsequently the rotation speed of a rotor by means of a laser tachometer is recorded. Third, owing to the produced lift, the indicated weight decreased. Fourth, lift force is estimated accurately by subtracting new weight from the initial weight at various rotational speeds of the propeller. Ultimately, dynamic pressure at the certain points on the fuselage is measured by a pitot tube, which is connected to an AZ-82012 digital manometer with accuracy of \pm 0.01 and the range of 0 to 1 psi (Fig. 8). It shows the difference between total and static pressure; which is dynamic pressure. Using Bernoulli's equation (Eq. 3) the fluid flow velocity on the curvature of the body is simply obtained,

$$
\left(\mathrm{P}_{s}+\rho \frac{V^{2}}{2}\right)=\mathrm{P}_{t} \Rightarrow V^{2}=\frac{2\left(\mathrm{P}_{t}-\mathrm{P}_{s}\right)}{\rho}
$$

Where $\rho$ is the density in $\mathrm{kg}$ per cubic meter, $\mathrm{V}$ is the fluid flow velocity in meter per second, Ps is the static pressure, and $\mathrm{Pt}$ is the total pressure in Pascal.

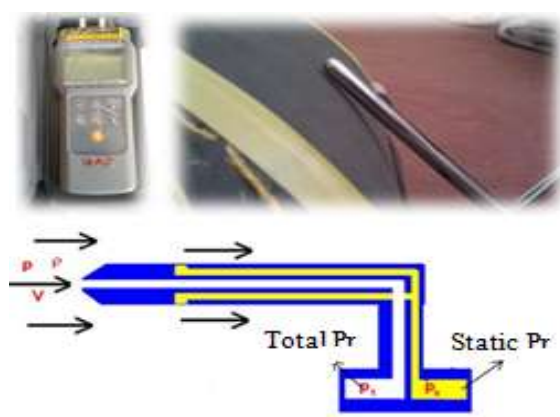

Fig.8 Digital manometer and pitot tube.

\section{Experimental Results}

In Fig. 9, the comparison of the lift of the Coanda aircraft with and without the axial vane is shown. As is clear, increasing the propeller speed yields growing the slope and the differences of the curves, which expresses that at the higher speeds, the axial vane is more effective. This effect is as a result of raising the speed of axial vane, which probably leads to producing Magnus effect and in addition, more suction in the above region of the axial vane appears, which finally generates significant additional lift. Indeed, by increasing the rotating speed of the motor, the flow speed goes up, as a result Coanda effect and lift shoots up. As mentioned before dynamic pressure was measured at the points which are clarified in Fig. 10 using pitot tube. Figure 11 depicts dynamic pressure versus the curvature of the aircraft body.

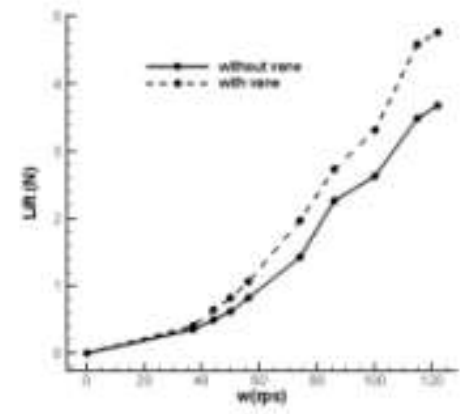

Fig.9 Lift of UAV with and without the axial vane versus engine rotating speed.

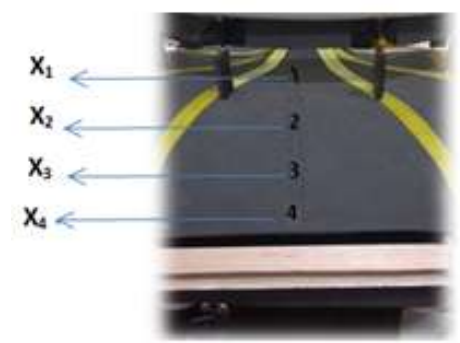

Fig.10 Points at which pressure dynamic was measured. 


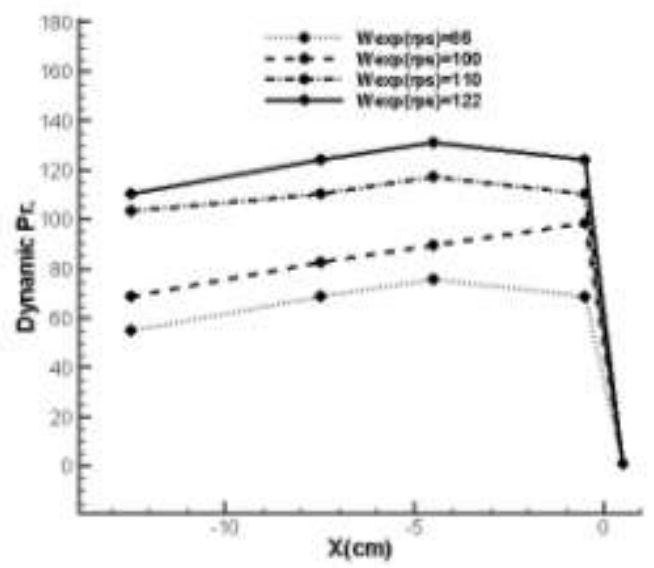

Fig.11 The dynamic pressure on the body with the axial vane.

\section{Numerical Analysis}

In this part, incompressible flow over the body of the Coanda UAV with and without the axial vane is investigated numerically. Geometry of the numerical simulations is similar to the experimental tests and there for the results are comparable to each other. Origin of the coordinate system is the location of the separation point where the vane is installed on the fuselage. $\mathrm{X}$ and $\mathrm{Y}$ axes are in the horizontal and vertical directions respectively. Two-dimensional simulations have been performed.

Figure 12 gives an overview of the computational domain. The border which represents the area around the body should be placed far enough in such a way that the flow pattern be independent of the boundary. To achieve this, the external boundary is selected a semi-circle with a radius of 13 times of the UAV height. Unstructured grid is employed to create the computational domain of the axial vane (Fig. 14). As could be seen from the Fig. 12, grid size near the body is small and far the wall is coarse, which reduces computational cost.

To simulate the fluid flow around the body with the axial vane at the separation point, domain consists of a rotating zone, including vane, and a stationary zone. Stationary and rotating regions are separated through a fluid-fluid interface to conserve continuity. In this situation, the interface between zones is defined interface boundary condition, and solutions are interpolated between two domains.

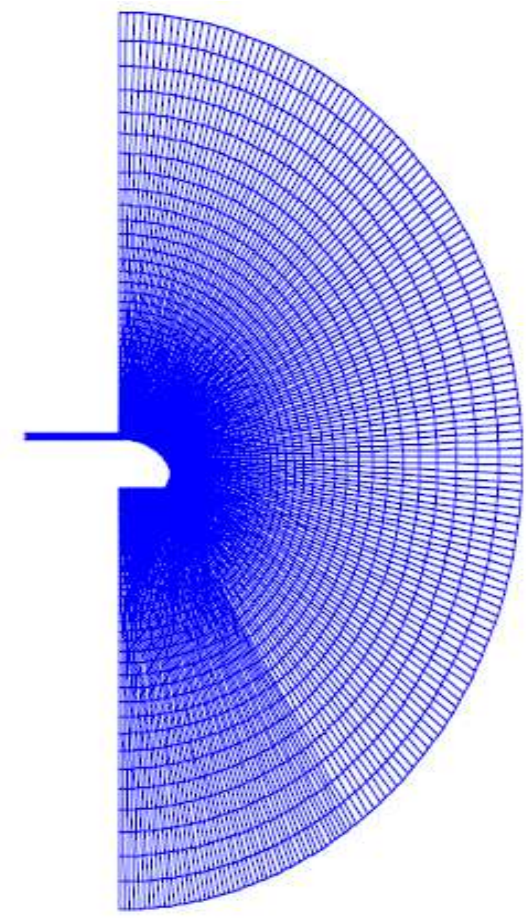

Fig.12 Overall view of the computational domain. 
Boundary conditions are shown in Fig. 13. A rectangular domain with the aspect ratio of 1:20 has specified as the entrance of the domain, hence fully developed turbulent flow in the gap exists. Velocity inlet is specified as an inlet boundary. According to experimental tests in this study, the velocity at this position at the moment of takeoff is approximately $15 \mathrm{~m} / \mathrm{s}$. V-velocity component is zero. No-slip condition is set at solid walls. Constant pressure boundary condition is selected for the outlet boundaries, which is equal to the ambient pressure.

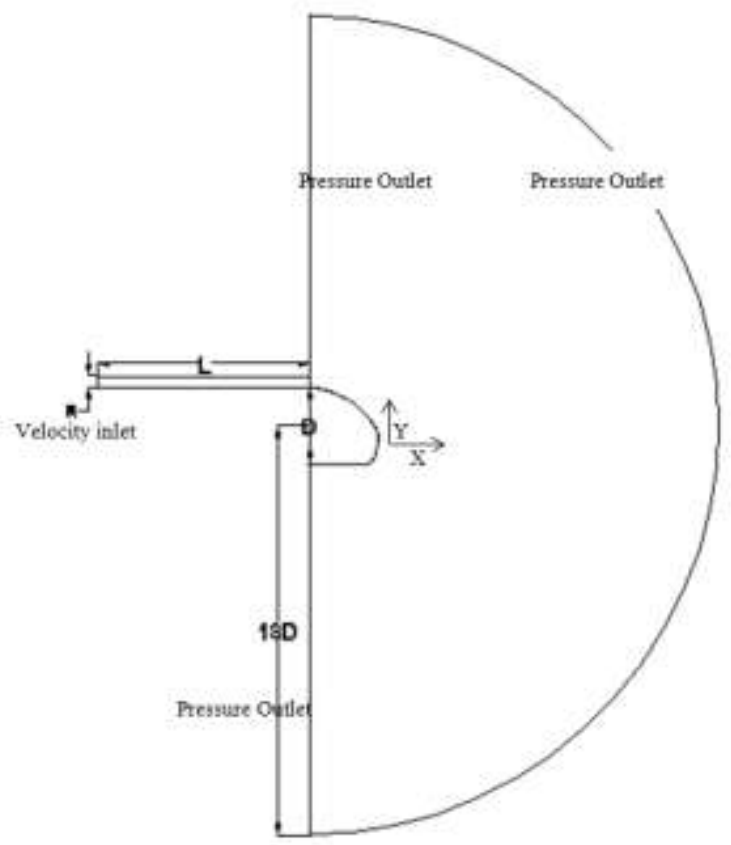

Fig.13 Geometry and boundary conditions.

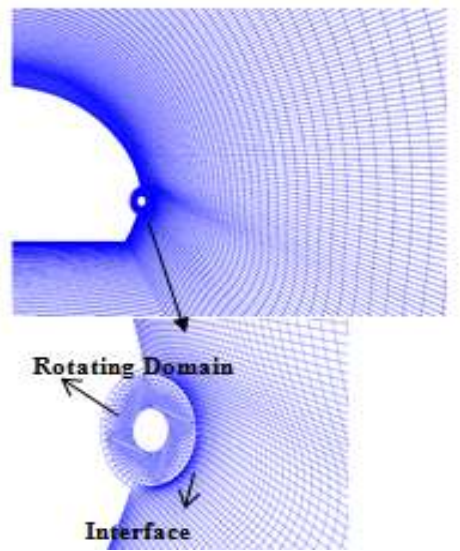

Fig.14 Mounted vane on the fuselage of the body.

SST (Shear Stress Transport Model) is a two equation turbulence model that combines the advantages of K- $\omega$ in the inner region of the boundary layer and K- $\varepsilon$ in the free shear flow [13]. This ability to switch between these models makes it an appropriate model to simulate this problem. An iterative solution algorithm, SIMPLE, is adopted for pressure-velocity coupling in steady state form. Second order upwind scheme is used to discretize incompressible Navier-Stokes equations, energy equation is omitted.

The laser tachometer has estimated the axial vane rotational speed in the range of $127 \mathrm{rev} / \mathrm{s}$ to $135 \mathrm{rev} / \mathrm{s}$ while the speed of motor was $122 \mathrm{rev} / \mathrm{s}$. consequently; the speed of the axial rotator was chosen $130 \mathrm{rev} / \mathrm{s}$ for numerical simulations. It was observed that at this rotational speed, the flow patterns of numerical simulations and experimental tests are analogous to each other. The flow separation occurs on the body thus fluid flow could not reach to the UAV's bottom part. Considering the axial vane speed, $130 \mathrm{rev} / \mathrm{s}$, and the diameter of the vane, 4 centimeter, the velocity of the fluid flow in proximity of the separation point is almost $2.5 \mathrm{~m} / \mathrm{s}$, which is reasonable. 


\section{Analyzing the flow and static pressure on the body of the Coanda UAV}

Figure 15 depict pressure contour of the model without the axial vane. Flow on the surface of the body is partly attached and follows the curvature of the surface. Pressure drop on the aircraft fuselage is responsible for production of lift, which is $7.27 \mathrm{~N}$.
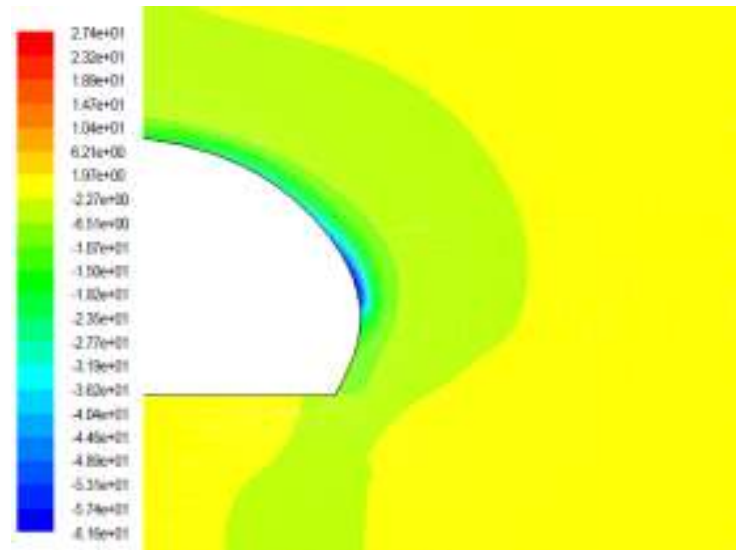

Fig.15 Static pressure contour of the basic model.

The axial vane rotates due to the flow around the curved part of the fuselage at the separation point. Figure 16 show pressure contours with the axial vane. In these figures, a vortex could be seen on the axial vane (Fig.17). Rotating vane creates a suction, which contributes to increase in lift about $1.52 \mathrm{~N}$.

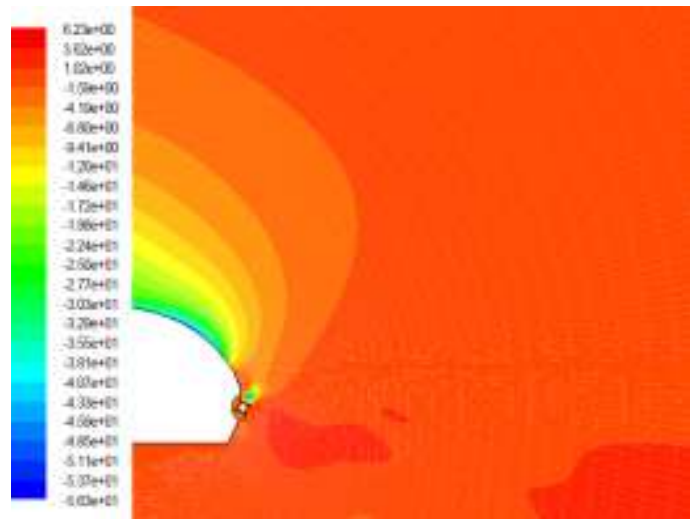

Fig.16 Static pressure contour of the UAV with mounted vane.

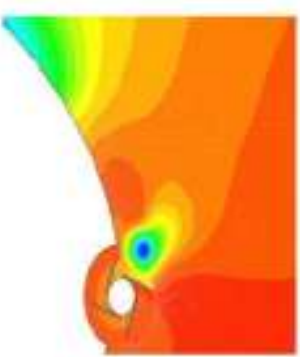

(a)

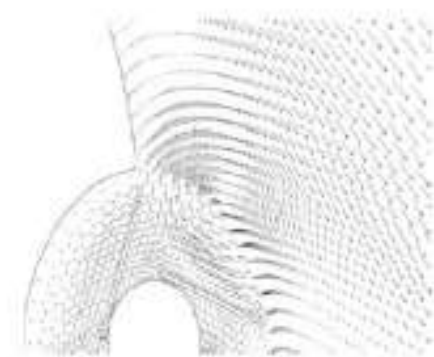

(b)

Fig.17 The static pressure contour of the axial vane(a) -formation the vortex above the axial vane and stream lines(b)

Pressure coefficient is a significant dimensionless number in fluid mechanics, which is the static pressure to dynamic pressure ratio in incompressible flows. Figure 18 shows pressure coefficient distribution along curved part of the body with and without the axial vane. The Axial vane raises negative pressure that leads to more Coanda effect and then additional lift. Results show that lift is increased $21 \%$ while the axial vane is installed at the separation point. Therefore, installed axial vane on the body at the point of separation has a favorable influence on Coanda effect, which means more lift. 


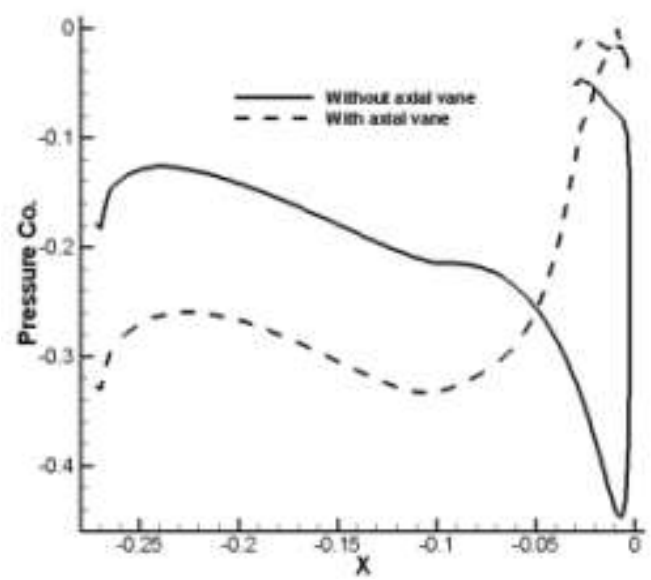

Fig.18 The pressure coefficient distribution on the body of the UAV in both cases, without and with axial

\section{Comparison Between Numerical And Experimental Results}

The impact of installing an axial vane on the body on lift has been evaluated experimentally. Results show that the axial vane has a remarkable positive influence on lift. Results of the numerical analysis of twodimensional model in both cases were compared to each other. It was revealed that mounting the axial vane at the separation point enhances lift.

In Fig. 18, the dynamic pressure distributions on the body with and without the axial vane experimentally and numerically are shown. There is a similarity between experimental and numerical dynamic pressure distribution. Both have the same trend when the rotational speed of propeller is $122 \mathrm{rev} / \mathrm{s}$. The maximum difference between numerical and experimental dynamic pressure is almost 100 Pascal that probably caused by the following reasons:

A) Numerical analysis are absolutely two-dimensional. However, three-dimensional effects are not insignificant. Therefore, ignoring the third dimension contributes to the differences between the results.

B) Undoubtedly, not simulating the real jet of the propeller has a significant adverse effect on the results.

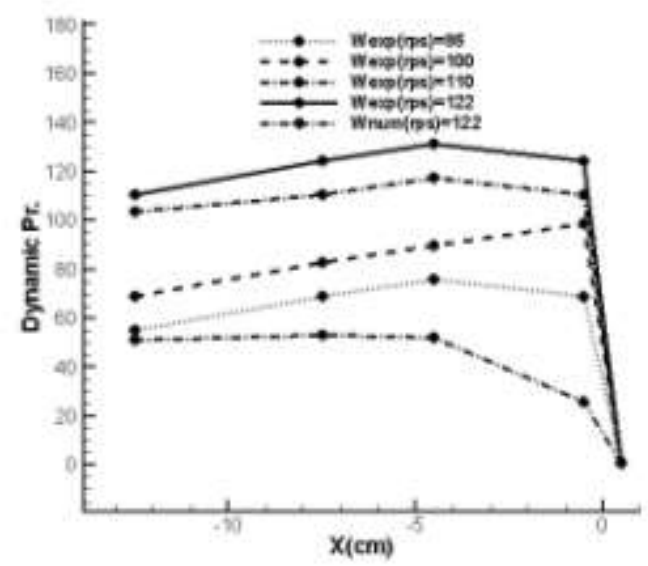

Fig.19 Comparison of experimental and numerical dynamic pressure at certain points on the body of Coanda UAV with the axial vane.

\section{Conclusion}

In the present study, the effect of mounting an axial vane on the body of the NO1 Coanda UAV at the separation point on lift has been investigated numerically and experimentally. The UAV was constructed and the separation point on the fuselage through tufts flow visualization technique was determined. Experimental results show $16 \%$ rise in lift when the axial vane is installed, and the rotational speed of the propeller is 122 rev/s. Furthermore, numerical simulations reveal that with the installation of the axial vane, the pressure coefficient distribution on the body is substantially reduced and subsequently lift increases. The results demonstrate that the axial vane leads to increase in lift from 7.27 to 8.79 Newton. The 21 percent growth in lift is remarkable. 
Comparison of numerical and experimental results illustrates that the trend of pressure coefficient distributions on the UAV's curvature is similar to each other, although they are not identical.

Growth of the lift of the UAV with the axial vane caused by the following reasons:

A) The reduction of pressure coefficient can be due to the pressure drop resulted from the rotating axial vane. The Magnus effect can also influence the rise in lift.

B) As can be seen from the static pressure contours (with and without vane), the rotating axial vane might create a vortex at the top of the vane, which induced a suction that results in improving the Coanda effect.

Research on optimizing the aerodynamic shape of the UAV's body to accomplish the maximum possible lift is a significant issue, which is worth to be considered. Three-dimensional numerical simulations with the axial vane are advantageous to compare with experimental results. Moreover, numerical and experimental studies to discover the optimal axial vane to install on the body in order to maximize lift are suggested.

\section{References}

[1] Barlow, C., Lewis, D., Prior, S.D., Odedra, S., Erbil, M., Karamanoglu, M., Collins, R., Investigating the Use of the Coandă Effect to Create Novel Unmanned Aerial Vehicles, In International Conference on Manufacturing and Engineering Systems Proceedings, number ISSN 2152-1522, 2009, pages 386-391.

[2] Feng, G., To Investigate Magnus Effect with Computational Fluid Dynamics Analysis, Dublin Institute of Thechnology, Bachelor, School of Mechanical and Design Engineering, Dublin Institute of Technology, Dublin, Ireland., 2009.

[3] Lift of Rotating Cylinders., "NASA Glenn Research Center", available at: https://www.grc.nasa.gov/www/K12/airplane/cyl.html.,2015.

[4] Nedelcut, F., Coanda Effect Uav-a New Born Baby in the Unmanned Aerial Vehicles Family, Review , Romania ministry of defencehenricoanda air force academy, 2010, pp. 21-28.

[5] Mirkov, N., and Rasuo, B., Numerical simulation of air jet attachment to convex walls and applications , Proceedings of 27th International Congress of the Aeronautical Sciences-ICAS, 2010.

[6] Dumas, A., Subhash, M., Trancossi, M., and Marques, J. P., The Influence of Surface Temperature on Coanda Effect, Energy Procedia Vol. 45, 2014, pp. 626-634.doi: 10.1016/j.egypro.2014.01.067, 2014.

[7] Inthasuwan, V., Prior, S. D., and Collins, R., An optimization study of Coandã wall jet phenomena , Innovation, Communication and Engineering,2013, pp. 437-440.

[8] Geoffrey, H., and Simon, M., "Aerofoil surface for controlling spin", Google Patents, 2006.

[9] Haixin, C., and Yu, S., Numerical Experiments on the Lift Generating Mechanisms of the GFS UVA ,44th AIAA/ASME/SAE/ASEE Joint Propulsion Conference \& Exhibit. American Institute of Aeronautics and Astronautics.doi:10.2514/6, $2008-4593$.

[10] Yifei, Z., Lijun, X., and Haixin, C., Numerical Research on Aerodynamic Efficiency of a VTOL GFS UAV , 53rd AIAA Aerospace Sciences Meeting. American Institute of Aeronautics and Astronautics.doi:10.2514/6, 2015-1678.

[11] Naudin. J.L., The GFS-UAL Model N-01a ,available at: http://jlnlabs.online.fr/gfsuav/gfsuavn01a.htm, 2006.

[12] Rae, W. H., and Pope, A. Low-speed wind tunnel testing (John Wiley, 1984).

[13] Wilcox, D. C., Turbulence modeling for CFD (DCW Industries, Inc., La Cãnada, CA, 1993). 